\title{
Chemical Fingerprinting and Chemical Analysis of Galactic Halo Substructure
}

\author{
Steven R. Majewski ${ }^{1}$, Mei-Yin Chou ${ }^{1}$, Katia Cunha ${ }^{2}$, Verne V. \\ Smith $^{2}$, Richard J. Patterson ${ }^{1}$ and David Martínez-Delgado ${ }^{3}$ \\ ${ }^{1}$ Dept. of Astronomy, University of Virginia, Charlottesville, VA 22904-4325, USA \\ ${ }^{2}$ National Optical Astronomy Observatories, PO Box 26732, Tucson, AZ 85726, USA \\ ${ }^{3}$ Instituto de Astrofisica de Canarias, La Laguna, Spain
}

\begin{abstract}
We present high-resolution spectroscopic measurements of the abundances of the $\alpha$ like element titanium (Ti) and s-process elements yttrium (Y) and lanthanum (La) for M giant candidates of (a) the Sagittarius (Sgr) dwarf spheroidal + tidal tail system, (b) the TriangulumAndromeda (TriAnd) Star Cloud, and (c) the Galactic Anticenter Stellar Structure (GASS, or Monoceros Stream). All three systems show abundance patterns unlike the Milky Way but typical of dwarf galaxies. The Sgr system abundance patterns resemble those of the Large Magellanic Cloud. GASS/Mon chemically resembles Sgr but is distinct from TriAnd, a result that does not support previous suggestions that TriAnd is a piece of the Monoceros Stream.
\end{abstract}

Keywords. galaxies: interactions, Galaxy: halo, Galaxy: structure, stars: abundances

\section{Results}

We obtained echelle spectra of M giant stars spatially and kinematically associated with the Sagittarius (Sgr), Triangulum-Andromeda (TriAnd) and Galactic Anticenter Stellar Structure (GASS) systems using the KPNO 4-m, ARC 3.5-m and TNG 3.6-m telescopes. The majority of Sgr stars show peculiar abundance patterns compared to those of nominal Milky Way (MW) stars (left panel, Fig. 1) and, as a group, form a coherent picture of chemical enrichment of the Sgr dwarf spheroidal (dSph) from $[\mathrm{Fe} / \mathrm{H}]=-1.4$ to solar abundance (Chou et al. 2007, 2009). The overall [Ti/Fe], [Y/Fe], [La/Fe] and $[\mathrm{La} / \mathrm{Y}]$ trends with $[\mathrm{Fe} / \mathrm{H}]$ for the Sgr stream + core stars resemble those seen in the Large Magellanic Cloud (LMC) and other dSphs, only shifted from them by $\Delta[\mathrm{Fe} / \mathrm{H}] \sim+0.4$ (LMC) and $\sim+1$ dex (dSphs), respectively (middle panel, Fig. 1); these relative shifts reflect the faster and/or more efficient chemical evolution of Sgr compared to the other satellites. By tracking the evolution of abundance patterns along the Sgr stream we can follow the time variation of the chemical make-up of dSph stars donated to the Galactic halo. This evolution demonstrates that while the bulk of the stars currently in the Sgr $\mathrm{dSph}$ are quite unlike those of the Galactic halo, an increasing number of stars farther along the Sgr stream have abundance patterns like metal poor MW halo stars, a trend that shows clearly how the Galactic halo could have been contributed by present day satellite galaxies even if the present chemistry of those satellites is now different from typical halo field stars. We also have analyzed the chemical abundances of a group of M giants found among the Sgr leading arm stars at the North Galactic Cap (NGC) but having radial velocities unlike the infalling Sgr leading arm debris there. Through "chemical fingerprinting" we conclude that these mostly receding northern hemisphere M giants are also Sgr stars - likely trailing arm debris overlapping the Sgr leading arm.

The origin of the recently identified GASS "ring", also known as the Monoceros Stream, remains controversial - e.g., whether it is truly a tidal stream or a part of the MW disk. 

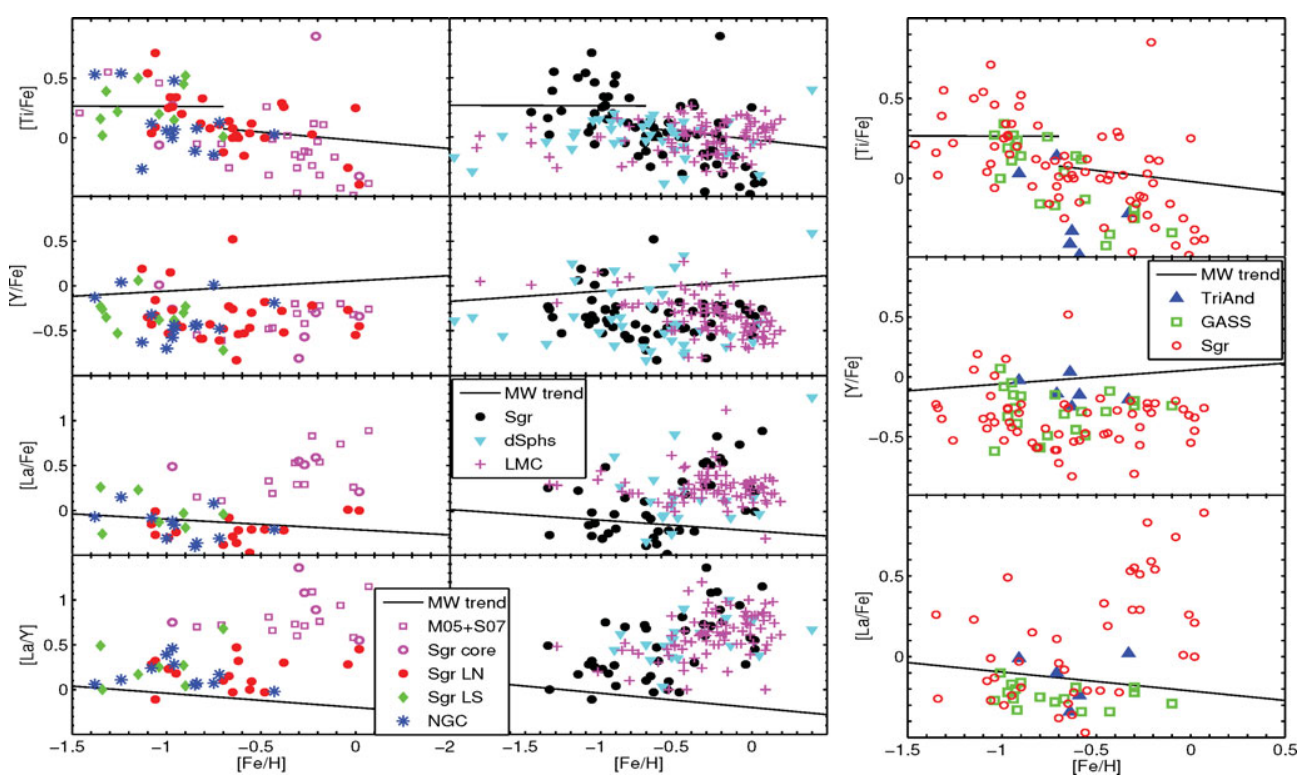

Figure 1. Left: $[\mathrm{Ti} / \mathrm{Fe}],[\mathrm{Y} / \mathrm{Fe}],[\mathrm{La} / \mathrm{Fe}]$ and $[\mathrm{La} / \mathrm{Y}]$ as a function of $[\mathrm{Fe} / \mathrm{H}]$. The open circles are giants from the Sgr core (including data from Monaco et al. 2005 [M05] and Sbordone et al. 2007 [S07], open squares), filled circles are from the Sgr leading arm in the northern hemisphere (LN), diamonds are from the leading arm in the southern hemisphere (LS), and asterisks are the "NGC" group. The solid line is a fit to the Galactic trend for halo and disk field stars (see discussion in Chou et al. 2009). Middle: Same as left, but comparing Sgr (circles) with the LMC (crosses) and other dSphs (triangles), after applying a shift of +0.4 dex in $[\mathrm{Fe} / \mathrm{H}]$ for LMC and +1 dex in $[\mathrm{Fe} / \mathrm{H}]$ for other dSphs. All LMC and dSph data are taken from the literature see Chou et al. (2009). Right: The distribution as a function of $[\mathrm{Fe} / \mathrm{H}]$ of the abundance ratios of $[\mathrm{Ti} / \mathrm{Fe}],[\mathrm{Y} / \mathrm{Fe}]$ and $[\mathrm{La} / \mathrm{Fe}]$ for TriAnd stars (triangles), GASS stars (open squares) and Sgr stars (open circles).

We find that the Ti and s-process abundances of GASS stars are similar to those of Sgr stars (right panel, Fig. 1) - consistent with the notion that GASS stars formed in the chemical environment of a dwarf galaxy that subsequently disrupted. However, differences in $[\mathrm{La} / \mathrm{Fe}]$ at high $[\mathrm{Fe} / \mathrm{H}]$ suggest that the GASS progenitor may have enriched faster than Sgr, which evolved at a rate slow enough to allow the yields of low metallicity AGB stars to enrich its younger populations.

Contrary to the proposal of Peñarrubia et al. (2005) that TriAnd is a distant part of the Monoceros Stream, we find that, although the chemical patterns of TriAnd are characteristic of other dwarf galaxies that contain metal-rich $\mathrm{M}$ giant stars, the TriAnd patterns are different enough from those of GASS/Mon specifically (especially [Y/Fe]; right panel, Fig. 1) to suggest that the TriAnd Star Cloud is likely an independent halo substructure unrelated to the GASS/Monoceros Stream.

\section{References}

Chou, M.-Y., et al. 2007, ApJ, 670, 346

Chou, M.-Y., et al. 2009, ApJ, submitted

Monaco, L., et al. 2005, A\&SA, 441, 141

Peñarrubia, J., et al. 2005, ApJ, 626, 128

Sbordone, L., et al. 2007, A\&AA, 465, 815 